\title{
The Influence of Ceramic Far-Infrared Ray (cFIR) Irradiation on Water Hydrogen Bonding and its Related Chemo-physical Properties
}

\section{Leung TK ${ }^{1,2,3 *}$, Lin $\mathrm{SL}^{4}$, Yang $\mathrm{TS}^{5}$, Yang $\mathrm{JC}^{5}$ and Lin $\mathrm{YS}^{6}$}

${ }^{1}$ Department of Physics, Fu Jen Catholic University, Hsinchuang, Taiwan

${ }^{2}$ Department of Diagnostic Radiology, Taipei Municipal Wanfang Hospital \& Department of Radiology, School of Medicine, College of Medicine, Taipei Medical University, Taiwan

${ }^{3}$ Diagnostic Radiology department, Taipei Hospital, Ministry of Health and Welfare, Taiwan

${ }^{4}$ Department of Surgery, Taipei Hospital, Ministry of health and Welfare, No. 117, Su Yuan Road, Hsinzhuang, New Taipei City 242-13, Taiwan

${ }^{5}$ School of Dental Technology, Taipei Medical University, Taipei 110, Taiwan

${ }^{6}$ Department of Applied Cosmetology and Graduate Institute of Cosmetic Science, Hungkuang University, Taichung, Taiwan

\begin{abstract}
The property of water is highly related to the earth's environment and climate change. The fundamental dynamical process of water is include formation and breaking of hydrogen bonds. This dynamic process, so far, is still poorly understood. We investigated weakening of the hydrogen bonds of water after ceramic Far-Infrared Ray (cFIR) irradiation and the resulting effects on physical and chemical properties of water. In this study, the Fourier transform infrared spectroscopy (FT-IR) was used to explore hydrogen bonding change of cFIR-irradiated water; in addition, capillary viscometers, Gas Chromatographs (GC), Differential Scanning Calorimetry (DSC), contact angles, Franz cells, High-Performance Liquid Chromatography (HPLC), and capillary electrophoresis analysis were used to evaluate its physical characteristics, such as viscosity, volatility, temperatures of water crystallization, surface tension, diffusion, hydrogen peroxide dissociation, solubility of solid particles, and changes in $\mathrm{pH}$ of acetic acid. The cFIR treated water decreased in viscosity and surface tension (contact angles), but increased in the solubility of solid particles, hydrogen peroxide dissociation, temperatures of water crystallization, and acidity of acetic acid The weakening of water hydrogen bonds caused by cFIR irradiation is correspondent with our previous medicalbiological studies on CFIR.
\end{abstract}

Keywords: Contact angle; Ceramic far infrared ray (cFIR); Irradiation water; Fourier transform infrared spectroscopy (FT-IR); Hydrogen bonds; Solubility; Volatility

\section{Introduction}

Water possesses important properties required for life-giving processes. These properties are effectuated by a hydrogen-bonded environment particularly evident in liquid water. Each liquid water molecule is involved in approximately four hydrogen bonds with strengths considerably less than covalent bonds but considerably greater than natural thermal energy. These hydrogen bonds are roughly tetrahedrally arranged, such that when strongly formed local clustering expands, the density decreases. It is different from actual chemical bonds such as ionic and covalent bonds. Hydrogen bonding is at onetenth of the strength of normal covalent bonds within a molecule. Hydrogen bonding in water, combined with its tendency to form open tetrahedral networks at low temperatures, and possess its characteristic properties [1-9].

The cluster formations where all water molecules are linked by three or four strong hydrogen bonds. The characteristics of hydrogen bonding that are responsible for the special properties of water allow it to act in diverse ways under different conditions, such as the clustering of water molecules [10-12]. For example, Stronger hydrogen bonds decrease viscosity because they decrease the liquid's interactions between molecules (Table 1) [1,3-9,13,14].

By decreasing the size of water clusters, the physical properties of water, including permeability and diffusibility, may be improved $[1,2,15]$. Many other properties and characteristics of hydrogen bonding relating to the weakening effect of hydrogen bonds of water may also exist (Table 1).

Many methods have been used to reduce the size of water clusters, such as magnetic fields, electric fields, heat, and far-infrared ray (FIR) irradiation [13,14]. Previous studies demonstrated that FIR with wavelengths of 4-16 $\mu \mathrm{m}$ appears to non-specifically induce an increase in the temperature of body tissues and elevate the motility of body fluids because of decreases in the size of water clusters in living organisms $[1,2,15]$. Researchers have deduced, but lack of strong evidence, that FIR could break the intermolecular hydrogen bonds by exciting stretching or bending vibrations in water clusters, and reducing the size of water clusters [10-12]. Ceramic material irradiate far infrared

\begin{tabular}{|l|l|}
\hline Property & Weakening Hydrogen bond \\
\hline Viscosity & Decrease \\
\hline Solubility of solid particles & Increase \\
\hline Water cluster frequencies (cluster size) & Decrease (cluster size decrease) \\
\hline Volatility & Increase \\
\hline Diffusion Coefficient & Increase \\
\hline Surface tension & Decrease \\
\hline
\end{tabular}

Table 1: Potential changes in the properties of weakening hydrogen bonds of liquid water.

*Corresponding author: Leung TK, Department of Physics, Fu Jen Catholic University, No.510, Zhongzheng Rd., Xinzhuang Dist., New Taipei City 24205, Taiwan (R.O.C), Tel: (886) 2-89255261; E-mail: hk8648@tmu.edu.tw

Received February 27, 2014; Accepted July 25, 2014; Published August 05, 2014

Citation: Leung TK, Lin SL, Yang TS, Yang JC, Lin YS (2014) The Influence of Ceramic Far-Infrared Ray (cFIR) Irradiation on Water Hydrogen Bonding and its Related Chemo-physical Properties. Hydrol Current Res 5: 174. doi:10.4172/2157 7587.1000174

Copyright: @ 2014 Leung TK, et al. This is an open-access article distributed unde the terms of the Creative Commons Attribution License, which permits unrestricted use, distribution, and reproduction in any medium, provided the original author and source are credited. 
Citation: Leung TK, Lin SL, Yang TS, Yang JC, Lin YS (2014) The Influence of Ceramic Far-Infrared Ray (cFIR) Irradiation on Water Hydrogen Bonding and its Related Chemo-physical Properties. Hydrol Current Res 5: 174. doi:10.4172/2157-7587.1000174

Page 2 of 10

ray under room temperatures (cFIR) was developed and applied in our laboratory, which was proven to have various biological and medical effects [16-25]. However, its fundamental physical-biological mechanism has not been fully explored. Various tests were performed to investigate its effects on the hydrogen bonding of liquid water.

\section{Materials and Methods}

\section{cFIR ceramic powder}

The FIR-emitting ceramic powder is micro-sized particles composed of numerous elemental ingredients, including calcium $(\mathrm{Ca})$, zirconium $(\mathrm{Zr})$, sulfur $(\mathrm{S})$, silicon $(\mathrm{Si})$, aluminum $(\mathrm{Al})$, magnesium $(\mathrm{Mg})$, iron $(\mathrm{Fe})$, oxygen $(\mathrm{O})$, and carbon $(\mathrm{C})$, which were developed by the biomaterials laboratory of Taipei Medical University, Taiwan [1625]. A Scanning Electron Microscopy (SEM) image on the cFIR powder was demonstrated in this study (Figures $1 \mathrm{a}$ and $1 \mathrm{~b}$ ), and emitted a high infrared ray with the emissivity at 4 to $16 \mu \mathrm{m}$; the ratio of radiation energy irradiated from the sample to an ideal black body as described by the Planck's Law was determined by a CI SR5000 infrared spectroradiometer (CI, Ltd., Migdal HaEmek, Israel).

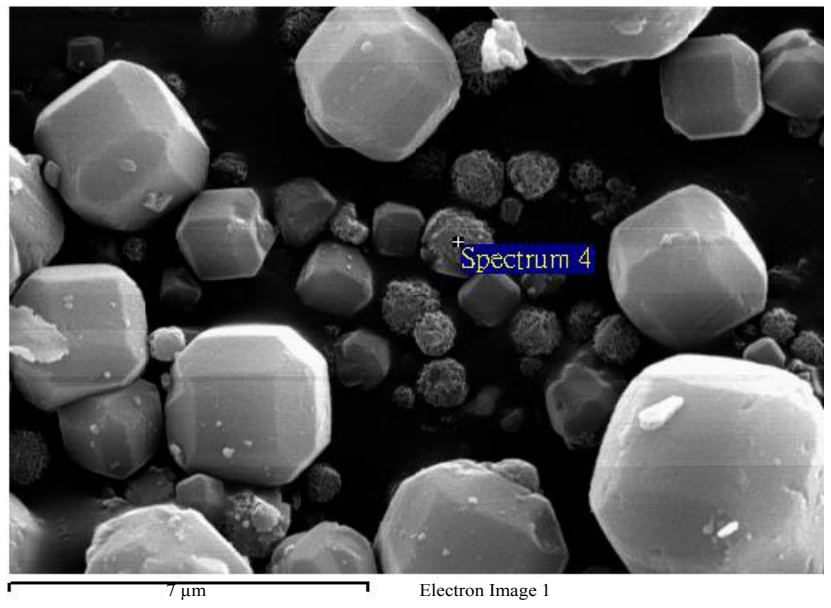

Figure 1a: Electronic microscopic image of the cFIR powder.

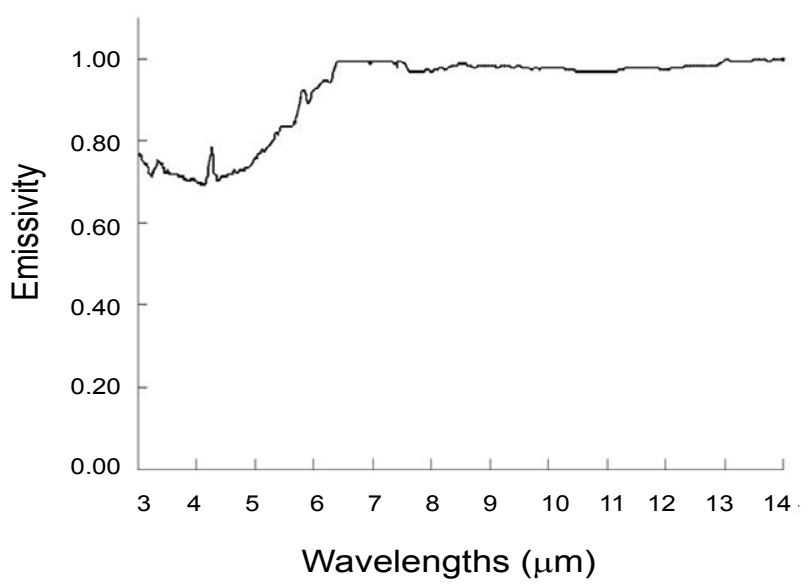

Figure 1b: The range of wavelengths representing FIR is from 8 to approximately $14 \mu \mathrm{m}$, and the average emissivity of the BIOCERAMIC powder is averaged over .90

\section{Fourier transforms infrared spectroscopy (FT-IR) measure-} ment

The non-cFIR double distilled water (DDW) and cFIR-irradiated DDW treated for $1 \mathrm{~h}$ was prepared for the infrared measurement. The liquid specimen was sealed between a pair of $\mathrm{CaF}_{2}$ windows (Beckman FH-01) with a thin Teflon spacer approximately $150 \mathrm{~nm}$ in thickness. Infrared spectrum was recorded at $25^{\circ} \mathrm{C}$ using a Nicolet Magna 550 spectrometer equipped with a dTGS detector. Each spectrum was recorded at a resolution of $2 \mathrm{~cm}^{-1}, 32$ scans was carried out and merged for each sample. The data were then calculated from absorption spectrum in the FIR region $\left(3400 \mathrm{~cm}^{-1}\right)$.

\section{Fluid viscosity test by an optically trapped Brownian particle}

Recently, we have presented an image-based approach for a nonintrusive fluid viscosity measurement in micro domains [26,27]; details of this system has been described previously [27]. We applied optical tweezers to control the position of optically trapped bead in confined region and use fluctuation of the trapped bead as a micro domain sensor to probe the local viscosity. Here, the two-dimensional mean square displacement (2-D MSD) was applied to capture the characteristics of the motion of a Brownian particle trapped by optical tweezers, where MSD is proportional to diffusion coefficient (D) in diffusive Brownian motion regime, namely, $\mathrm{MSD}=4 \mathrm{D} \Delta \mathrm{t}[28]$ and diffusion coefficient is proportional to $(T / \eta)$, where $\Delta t, T$ and $\eta$ are time lag, temperature and temperature-dependent fluid viscosity, respectively. This approached were further applied to evaluate the possible effect of cFIR on fluid viscosity. In this study, the optical tweezers system was applied to trap polystyrene bead $(1.87 \mu \mathrm{m}$, Bangs Laboratories, Fishers, IN), where sequential images of the trapped bead were acquired by the EMCCD camera (Luca ${ }^{\mathrm{EM}}$ DL6581, Andor) and were used to track the fluctuation of an optically trapped bead in a fluid with or without the effect of cFIR (Figure 2).

\section{Viscosity test by a capillary viscometer}

The effects of the cFIR irradiation on the intra-molecular hydrogen bonding of water were indirectly assayed by a capillary viscometer. Figure 3 show the specimen received the cFIR treatment, setup by 20 $\mathrm{ml}$ aliquot DD water for $1 \mathrm{~min}$ at ambient temperature. The kinematic viscosity $(v)$ was measured by a calibrated Cannon-Ubbelohde capillary viscometer (Schott, 532 03/ 0C, Germany) with a control unit (Lauda, PVS1-X02004, Germany). The viscometers were placed inside the thermostat with a water bath (Lauda, E200, Germany) at $25^{\circ} \mathrm{C}$. Data were correlated using the statistical analysis system (Lauda DR. R. WOBSER GMBH \& CO.) package. Kinematic viscometry is defined as the quotients of the dynamic viscosity by the density $v_{k i n}=\frac{\eta}{\rho}$ and has the unit $\mathrm{mm}^{2} / \mathrm{s}$ (or centistokes, cst). Kinematic viscosities $v$, expressed in centistokes, were calculated from the measured flow time $\theta$ and instrument constant $\mathrm{c}$ by using the following equation: $v=c \theta$. The values for $\mathrm{c}$ are provided by the viscometer manufacturer. The viscometer constants were calibrated for effects of temperature.

\section{Volatility}

GC-SPME analysis of the sorghum wine after direct cFIR irradiation: Samples of 58\% sorghum wine were mixed with the cFIR ceramic powder $(20 \mathrm{~g})$ and irradiated for $20 \mathrm{~min}$ in a sealed glass vessel. Control samples were prepared following the same procedures as the experimental group, and placed in an isolated system for $20 \mathrm{~min}$ without irradiation. Sample volatility was tested using the Gas Chromatograph (GC) analyses of the solid-phase micro-extraction (SPME) fractions, using a Varian STAR 3400Cx series The mobile phase ratio was acetonitrile- 




Figure 2: Schematic diagram of the optical tweezers system with the image acquisition arrangement for detecting Brownian motion before and after cFIR irradiation, where the laser beam is expanded fivefold with lens pairs (L1:L2) to slightly overfill the back aperture of the objective lens (Plan Apo 60x/1.40 oil, Nikon), and directed into objective lens via a dichroic mirror D1 (780dcspxr, Chroma). Here we apply Fourier-based cross-correlation processing to determine the lateral displacement of the optically trapped bead; therefore, both the mean square displacement (MSD) and the diffusion coefficient (D) can be obtained.

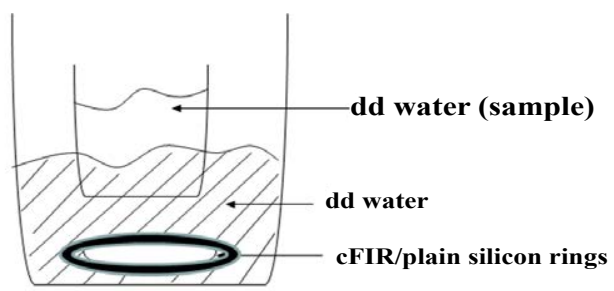

Figure 3: The schematic diagram for water irradiated by a silicon ring with or without CFIR powders.

water $(0.1 \%$ TFA) $80: 20$, and the flow rate was $1 \mathrm{ml} / \mathrm{min}$. Indomethacin was detected at a wave length of $320 \mathrm{~nm}$ [15].

II GC, equipped with a $30 \mathrm{~m} \times 0.5 \mathrm{~mm}$ I.D. and $0.25 \mu \mathrm{m}$ film thickness DB Waxter fused with a silica capillary column, connected to a Varian Saturn III mass selective detector using split less injectors. The initial oven temperature was set to $50^{\circ} \mathrm{C}(3 \mathrm{~min})$, and then increased to $200^{\circ} \mathrm{C}$, and was maintained at a constant temperature for $5 \mathrm{~min}$. The injector temperature was $240^{\circ} \mathrm{C}$, and the transfer line was maintained at $220^{\circ} \mathrm{C}$, using nitrogen $\mathrm{N}^{60}$ carrier gas with a column-head pressure of $13 \mathrm{psi}(1 \mathrm{psi}=6894.76 \mathrm{~Pa})$.

GC-SPME analysis of the sorghum wine after indirect cFIR irradiation: The $20 \mathrm{~g}$ power of cFIR ceramic, enclosed in plastic bags, was used to irradiate various water samples (10 $\mathrm{ml}$ in a glass dish) for $20 \mathrm{~min}$. Samples of $58 \%$ sorghum liquor $(2 \mathrm{ml}$ each) were irradiated by the cFIR-irradiated water samples (indirect contact separated by collection tubes) for $1 \mathrm{~min}$ in a closed system. The control samples were also prepared using the same procedure as the experimental groups but using usual water samples. GC analyses of the SPME experiments were also conducted using a Varian STAR $3400 \mathrm{Cx}$ series II GC. The following procedures and conditions were identical to those described in the previous section.

\section{Diffusion test model by passing drug (Indomethacin) through} the artificial membrane

Franz cell apparatus: Cellulose acetate membranes with a molecular weight (MW) cutoff of 10,000 (Spectrum, Laguna Hills, CA) were trimmed into circular discs (diameter approximately $10 \mathrm{~mm}$ ) sufficient to cover the effective diffusion area of the receptor, and then soaked in a phosphate buffer ( $\mathrm{pH} 7.4)$ for at least $12 \mathrm{~h}$. Cellulose acetate membranes, which contained glycerin, were rinsed once with the receptor fluid before placing them onto the receptor. Membranes were hydrated and sandwiched between two microscope glass slides and submerged in receptor fluid in a Petri dish to prevent creases or folds when wetted. Air bubbles are trapped within the interface of membrane and receptor. The donor compartment was then covered and closed up tightly by a pinch clamp. During the operation of the Franz cell, we collected $500 \mu \mathrm{l}$ from the receptor buffer through a sample port by using a pipette [15]. Drug content was assessed by HPLC methods. HPLC analysis was performed using a Waters 2487 HPLC UV-Visible Detector (Milford, MA, USA) with an auto sampler equipped with a quaternary pump and a variable-wavelength detector. All samples were analyzed using a reverse phase C18 column (Microsorb-MV C18 15 $\mathrm{cm}, 5 \mu \mathrm{m})$. The mobile phase ratio was acetonitrile-water $(0.1 \%$ TFA) $80: 20$, and the flow rate was $1 \mathrm{ml} / \mathrm{min}$. Indomethacin was detected at a wave length of $320 \mathrm{~nm}$. 
Surface tension-measurement of water contact angles: We used a remote-computer-controlled goniometer system (Digidrop, GBX, France) to measure the contact angles. The cFIR-irradiated water and control water's contact angle to the surface were measured at $25^{\circ} \mathrm{C}$ and $70 \%$ relative humidity. A minimum of three readings were made on each sample, and the values from a minimum of 30 samples were averaged.

Analysis of Hydrogen Peroxide $\left(\mathrm{H}_{2} \mathrm{O}_{2}\right)$ dissociation - direct scavenging of $\mathrm{H}_{2} \mathrm{O}_{2}$ : An $\mathrm{H}_{2} \mathrm{O}_{2}$ solution (Sigma, St. Louis, MD, USA) with $1 \mathrm{M}$ concentration was prepared, and equal amounts of $9 \mathrm{ml} \mathrm{H}_{2} \mathrm{O}_{2}$ solution was added to the test tubes. $\mathrm{H}_{2} \mathrm{O}_{2}$ solution was categorized into two groups: the control and cFIR. For the cFIR group, test tubes were incubated at room temperature and externally covered by plastic bags filled with $100 \mathrm{gm}$ cFIR powder for $3 \mathrm{~h}$ of irradiation. The control group was treated similarly, with the exception of the cFIR treatment. After the incubation period, $7.5 \mathrm{mM}$ phenol red (Sigma, St. Louis, MD, USA) and $5 \mathrm{mg} / \mathrm{ml}$ horseradish peroxidase (Sigma, St. Louis, MD, USA) were added to each test tube. The mixture was allowed to react for $10 \mathrm{~min}$, and absorbance was observed at $550 \mathrm{~nm}$ by an enzyme-linked immune sorbent assay (ELISA) reader (Gemini XPS Molecular Devices, Sunnyvale, CA, USA), with lower absorbance values representing higher $\mathrm{H}_{2} \mathrm{O}_{2}$ scavenging ability.

Solubility-measured by capillary electrophoresis analysis: Water samples were prepared from ultra-pure water with $18.2 \mathrm{M} \Omega$ resistance (Satorius 337070, Germany). The water samples were preirradiated by FIR ceramic powder at room temperature for $20 \mathrm{~h}$, and the control water samples were covered with aluminum foil and stored in a dark environment at room temperature. After $20 \mathrm{~h}$, the water samples were aliquot into $25 \mathrm{ml}$ in the $50 \mathrm{ml}$ polypropylene centrifuge tubes. Aqueous extracts were prepared by infusing 0.1 grounded and homogenized dry green tea leaves into each of the $25 \mathrm{ml}$ water samples at room temperature for $10 \mathrm{~min}$. This was repeated five times for cFIR irradiation treatment for the statistic analysis. A thermal-controlled $\left(25^{\circ} \mathrm{C}\right)$ capillary electrophoresis (CE) system (G1600A, Agilent) was used and controlled by 3D-CE ChemStation software (Agilent) to obtain quantitative measurements of various components in the green tea samples. An untreated fused-silica capillary tube (Beckman; total length $48.5 \mathrm{~cm}$, effective length to detector $40 \mathrm{~cm}$, I.D. $50 \mu \mathrm{m}$, O.D. $375 \mu \mathrm{m}$ ) was thoroughly flushed with $1.0 \mathrm{~N} \mathrm{NaOH}$ for $50 \mathrm{~min}$ and then deionized water for $30 \mathrm{~min}$ before use. Before injection, the initialized capillary was preconditioned by rinsing sequentially with $1.0 \mathrm{~N} \mathrm{NaOH}$ for $2 \mathrm{~min}$, water for $1 \mathrm{~min}$, and a running buffer for $2 \mathrm{~min}$. The sample solution was injected at $30 \mathrm{mbar}$ for $5 \mathrm{~s}$, and then followed with an injection at $30 \mathrm{mbar}$ for $1 \mathrm{~s}$ of the running buffer to minimize the sample loss. The electrophoretic separation process was monitored with a built-in photodiode array (PDA) detector at 200, 205, 220, 266, and $280 \mathrm{~nm}$. After electrophoresis, the capillary was cleaned by flushing with deionized water for $2 \mathrm{~min}$.

Because of the better separation efficiency, better separation speed, and less consumption of water samples and reagent, CE has been used to be the complement to ion chromatography [29]. In this study, CE was used to verify the categories and the quantity of each catechin. A mixture of 13 main standards (include GC, caffeine, EGCG, EGC and EC) in tea was analyzed using the micellar electrokinetic capillary electrophoresis (MEKC) method [30].

Analysis of water crystallization temperature (Tc): Water samples treated by cFIR for 5 min were used as experimental group to contrast with control group in Tc analysis (DDW without cFIR treatment). About $5 \mathrm{mg}$ water sample was individually loaded into a volatile aluminum sample pan with cover and hermetically sealed. The specimen was placed in the chamber of the differential scanning calorimetry (DSC) apparatus (Model DSC2920, TA Instruments, Newcastle, Del., USA) and set the following procedures: cooling rate of $-5^{\circ} \mathrm{C} / \mathrm{min}$ from $40^{\circ} \mathrm{C}$ to $-40^{\circ} \mathrm{C}$, and then keep the temperature for $5 \mathrm{~min}$. The procedure was repeated 5 times in a dry nitrogen environment. The enthalpy of crystallization during cooling $\Delta \mathrm{Hc}$ was measured.

pH value determination of weak acid: Glacial acetic acid $(1 \mathrm{ml})$ was added to double-distilled (dd) $\mathrm{H}_{2} \mathrm{O}(9 \mathrm{ml})$ in a glass tube, to form a $10 \%$ acetic acid solution. After 2 min equilibration time, solution $\mathrm{pH}$ was measured and recorded. The glass tube was then covered with cFIR powder and irradiated for $2 \mathrm{~min}$, the solution $\mathrm{pH}$ was recorded, and changes in $\mathrm{pH}$ were noted.

\section{Statistical analysis}

Measurements were performed in multiple tests. Statistical evaluation between cFIR and control groups was determined by the paired $t$ test method, and data with a $p$ value less than .05 were considered significant.

\section{Results}

\section{Fourier transforms infrared spectroscopy (FT-IR)}

IR spectroscopy is one of the most precise methods to provide information on $\mathrm{H}$-bonds. In this experiment, we found the quantitative difference of cFIR-irradiated DDW and non-cFIR DDW in the FIR region $\left(3400 \mathrm{~cm}^{-1}\right)$, with a significant decrease in the absorbance of cFIR-irradiated DDW. This result indicates a hydrogen bond weakening effect of DDW after cFIR irradiation (Figure 4).

Because of the unusually strong absorption of liquid water in this mid-IR region, this spectrum of liquid water mainly consists of three types of vibrations of $\mathrm{H}_{2} \mathrm{O}$ molecules: the most intense $v_{\mathrm{s}}$ $(\mathrm{O}-\mathrm{H}$...) stretch band with a maximum at approximately $3400 \mathrm{~cm}$ ${ }^{1}$, the $\delta_{\mathrm{H}-\mathrm{O}-\mathrm{H}}$ bending band (vibration of the $\mathrm{H}-\mathrm{O}-\mathrm{H}$ angle) with the maximum at approximately $1640 \mathrm{~cm}^{-1}$, and the "librational" band $\rho_{\mathrm{H} 2 \mathrm{O}}$ at approximately $700 \mathrm{~cm}^{-1}$ (relative rotations of rigid $\mathrm{H}_{2} \mathrm{O}$ molecules, also called hindered rotations). The intra monomer bands ns and $\delta_{\mathrm{H}-\mathrm{O}-\mathrm{H}}$ correspond to those of water vapor.

\section{Fluid viscosity (by Brownian particle) or Effect of cFIR irradiation on fluid viscosity}

We then investigate the relationships between MSD and time lag

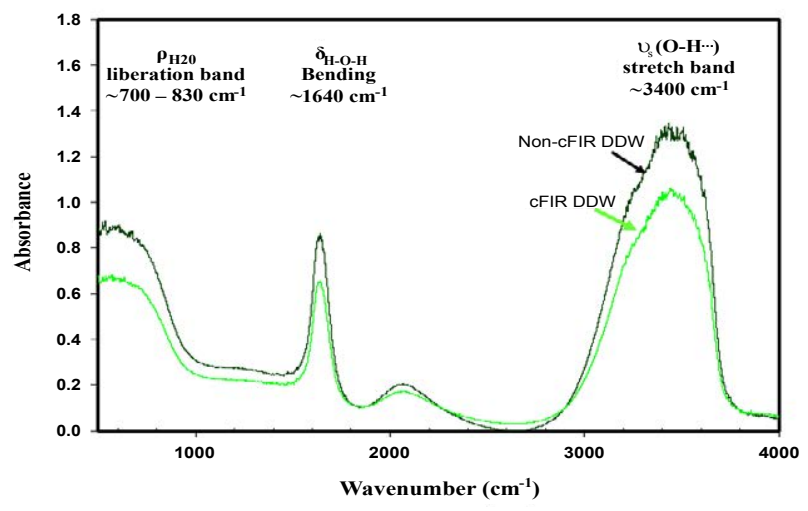

Figure 4: Significantly decreased absorbance area of cFIR-irradiated DDW in the FIR region $\left(3400 \mathrm{~cm}^{-1}\right)$ was noted, compared to the non-cFIR-irradiated DDW. 
Citation: Leung TK, Lin SL, Yang TS, Yang JC, Lin YS (2014) The Influence of Ceramic Far-Infrared Ray (cFIR) Irradiation on Water Hydrogen Bonding and its Related Chemo-physical Properties. Hydrol Current Res 5: 174. doi:10.4172/2157-7587.1000174

Page 5 of 10

$\Delta t$ to evaluate the possible effect of cFIR on fluid viscosity at room temperature. Figure 5 showed the MSD plots of an optically trapped polystyrene bead in a fluid with (cFIR irradiation for three minutes, solid symbol) or without the effect of Cfir (empty symbol). We find that MSD and time lag $\Delta t$ is linear within $\Delta t=40 \mathrm{~ms}$; however, significant deviations from linearity have been found at time intervals above $80 \mathrm{~ms}$ due to the optical confinement. This relation revealed that the motion of optically trapped bead showed a random walk during short time interval. In addition, as we mentioned earlier that diffusion coefficient (D $\Delta T / \Delta$ ) could be obtained from the linear slope of 2 -D MSD plot, namely, $\mathrm{MSD}=4 \mathrm{D} \Delta \mathrm{t}$, the present results showed that in short-time interval regime values of the linear slope of MSD plots in the experimental group (cFIR irradiation for three minutes) were larger than that in the control group (without cFIR irradiation), which implied cFIR irradiation decreased a fluid's viscosity, as can be seen in Figure 4. Note that the present result showed that change ratio of fluid's viscosity, $\left(\eta_{w / o}-\eta_{w /}\right) / \eta_{w / o}, \eta 0.12$, which implied fluid's viscosity is decreased to $12 \%$, where $\eta_{w / o}$ and $\eta_{w / \text { represent }}$ are fluid viscosity in the absence and presence of the effect of cFIR, respectively.

\section{Viscosity}

The flow time and kinematic viscosity of water samples determined by the capillary viscometer for the control water and the cFIRirradiated water were $300.37 \pm 0.91 \mathrm{~s}$ and $293.39 \pm 0.05 \mathrm{~s}$, and 0.92 $\pm 0.00 \mathrm{cSt}$ (centistokes) and $0.90 \pm 0.01 \mathrm{cSt}$, respectively. The flow time and viscosity reduction for water treated by cFIR irradiation was statistically significant from that of the control group, implying the weakening of intermolecular hydrogen bonding, respectively $(p<.01$; Table 4).

Volatility result of GC-SPME analysis of sorghum wine irradiated either directly or indirectly by cFIR ceramic materials

We determined changes in volatility of the cFIR-irradiated alcohol-

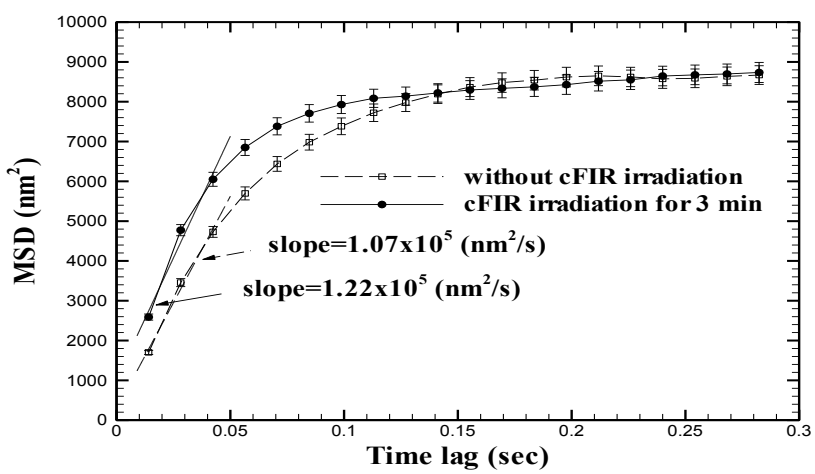

Figure 5: Effect of cFIR irradiation on fluid viscosity. MSD plots obtained from the optically trapped Brownian particle in a fluid with (cFIR irradiation for three minutes, solid symbol) or without the effect of cFIR (empty symbol) revealed that cFIR irradiation decreased fluid's viscosity. water mixture. Figure 6a shows elevation in ethanol volatility for the cFIR directly irradiated samples compared to the control group; Figure $6 \mathrm{~b}$ shows elevation in ethanol volatility for the indirect cFIR-irradiated samples compared to the control group, as measured by GC-SPME. The change in mean volatility of the cFIR groups, expressed as the total amount of ethanol extracted, was significantly higher than that of the control group $(p<.01)$.

\section{Result of diffusion model using drug (Indomethacin) pass through artificial membrane}

Figure 7 shows the transdermal diffusion of Indomethncin without and with cFIR irradiation in Franz cell experimental model. Under cFIR irradiation, the indomethacin solution was found significantly facilitated passed through the artificial skin membrane monitored by the Franz cell experimental model $(p<.01)$.

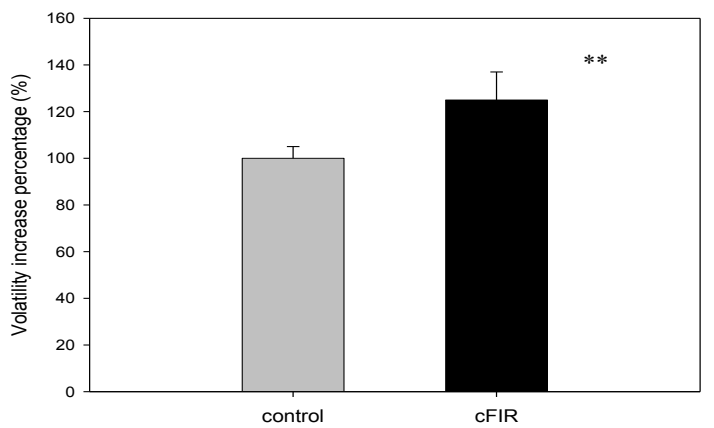

(a)



(b)

Figure 6: GC-SPME analysis for volatility changes in the sorghum liquor irradiated without and with directly cFIR irradiation (a) and indirectly cFIR irradiation through water samples. It which shows volatility increased of both directly cFIR irradiation (a) and indirectly cFIR irradiation in cFIR group (b). ${ }^{* *} p<.01$ is significantly different compared to the control group.

\begin{tabular}{|l|l|l|}
\hline $\begin{array}{l}\text { Physical relationship of hydrogen } \\
\text { bonds with water }\end{array}$ & Physical characteristics of the hydrogen bonds weakening & Possible applications \\
\hline Volatility & Weakening the hydrogen bonds and increasing the volatility & Consumption in industrial applications related to gas and liquid \\
\hline Diffusion & Weakening of the hydrogen bonds to enhance solubility & Chemical, food and pharmaceutical industry \\
\hline Solubility of solid particles & Weakening the hydrogen bonds can increase solid solubility & Applications in food \& cooking in dye/textile industries, etc. \\
\hline Temperature of crystallization & Weakening the hydrogen bonds can alter freezing temperatures & Application of freezing industry to save electricity \\
\hline pH change of weak acid & $\begin{array}{l}\text { Hydrogen bond on the acid-base increases the strength, and } \\
\text { cFIR enhances mild elevations in the acidity }\end{array}$ & Chemical, food, biochemical and pharmaceutical industry \\
\hline
\end{tabular}


Citation: Leung TK, Lin SL, Yang TS, Yang JC, Lin YS (2014) The Influence of Ceramic Far-Infrared Ray (cFIR) Irradiation on Water Hydrogen Bonding and its Related Chemo-physical Properties. Hydrol Current Res 5: 174. doi:10.4172/2157-7587.1000174

Page 6 of 10

\section{Surface tension}

The water contact angles of cFIR-irradiated water and control water are shown in Figure 8. The average results of cFIR-irradiated water had a lower contact angle than that of the control water. The

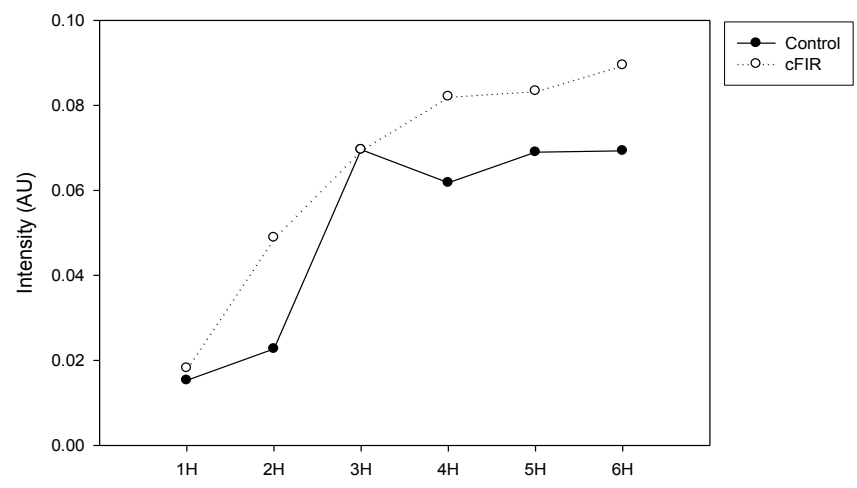

Figure 7: Shows significant increases of transdermal diffusion of Indomethancin under cFIR irradiation in the Franz cell experimental model. ( $Y$ axis: cumulative intensity $(\mathrm{AU})$ measured on indomethacin pass through artificial membrane; $\mathrm{X}$ axis: running per hour).

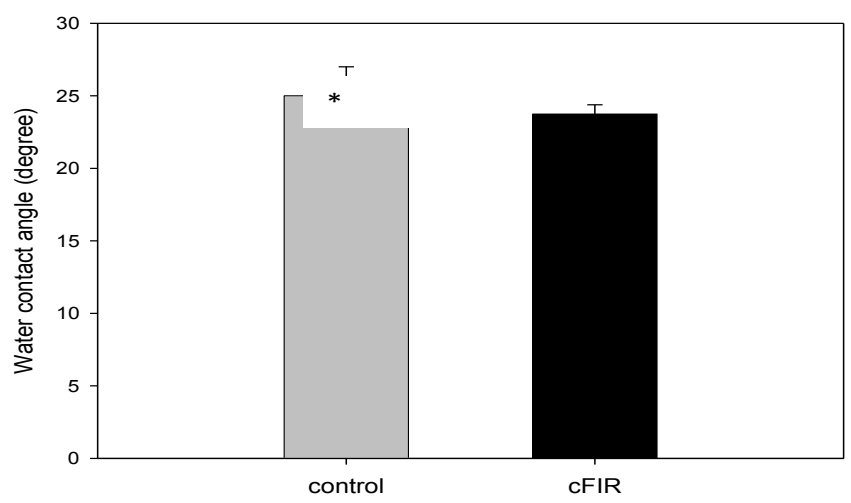

Figure 8: Comparison of water contact angle of the cFIR irradiated water and control water samples, which shows a significantly decreased compared with the control group $\left({ }^{*} p<.05\right)$.

\begin{tabular}{|l|c|c|c|}
\hline & cFIR treated DDW & DDW & P \\
\hline $\mathrm{Tc}\left({ }^{\circ} \mathrm{C}\right)$ & $-16.81 \pm 0.29$ & $-21.77 \pm 1.18$ & $.0010^{* *}$ \\
\hline $\mathrm{Tm}\left({ }^{\circ} \mathrm{C}\right)$ & $0.24 \pm 0.04$ & $0.08 \pm 0.04$ & $.0081^{* *}$ \\
\hline $\mathrm{DH}_{\text {fusion }}(\mathrm{J} / \mathrm{g})$ & $352.3 \pm 1.8$ & $288.0 \pm 31.2$ & $.0088^{* *}$ \\
\hline
\end{tabular}

The foot note is ** $\mathrm{P}<0.005$

Table 3: Thermal analysis results obtained from DSC thermograms of the water samples. Tc: crystallization temperature during cooling; Tm: melting temperature during heating.

\begin{tabular}{|c|c|c|c|c|}
\hline & \multicolumn{2}{|c|}{ Cummulative time of DDW indirectly irradiated by cFIR ring } & \multicolumn{2}{|c|}{ Cummulative time of DDW with plain silicon ring } \\
\hline & Time (sec) & Viscosity (cts) & Viscosity (cts) \\
\hline 1 & 293.38 & 0.9 & 299.97 \\
\hline 2 & 293.32 & 0.9 & 299.98 \\
\hline 3 & 293.43 & 0.9 & 299.79 \\
\hline 4 & 293.43 & 0.9 & 301.72 \\
\hline Average & $292.39 \pm 0.05$ & $0.90 \pm 0.00$ & 30.92 \\
\hline
\end{tabular}

Temperature: $25^{\circ} \mathrm{C}$; Capillary $\mathrm{K}$ value: 0.003086

Table 4: The viscosity reduction for water treated by cFIR irradiation was statically significant from that control group implying the weakening of intermolecular hydrogen bonding. change of surface tension may be the main reason for the different contact angle $(p<.05)$.

\section{Hydrogen peroxide dissociation}

Figure 9 shows that the mean absorbance of the control and cFIR groups found a significant decrease in the cFIR group $(\mathrm{n}=31)$. The extent of $\mathrm{H}_{2} \mathrm{O}_{2}$ disappearance in the cFIR group is larger than that in the control group with a $10.26 \%$ decrease. This result confirms that $\mathrm{H}_{2} \mathrm{O}_{2}$ can be scavenged directly by cFIR treatment.

\section{Solubility}

Results of cFIR irradiation on the green tea solution, measured by capillary electrophoresis (CE): In this study, CE was used to verify the categories and the quantity of each catechin. A mixture of 13 main standards (GC, caffeine, EGCG, EGC and EC) in tea was successfully analyzed by micellar electrokinetic capillary electrophoresis (MEKC). By comparing with the standard mixtures in the electropherogram, the constituents of green tea were identified. Figure 10 shows the separation profile of green tea prepared from two different water sources. The results show that these five peaks of green tea prepared by cFIR-treated water are higher than the control. Figure 10 also shows the comparisons of peak area analysis in these five major components (GC, caffeine, EGCG, EGC, and EC). Each component of green tea treated by cFIR is greater than the control. The average increase is $9.05 \%$ for the five representing constituents.

\section{Temperature of crystallization (Tc) determination}

The result of a DSC experiment is about heat flux versus temperature or versus time. There are two different conventions: exothermic reactions in the sample are shown with a positive or negative peak. The curves can be used to calculate enthalpies of transition of water.

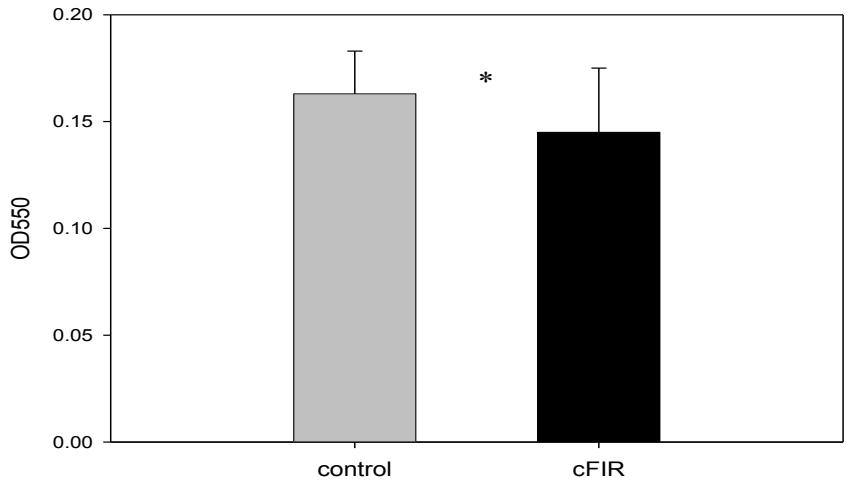

Figure 9: Comparison of $\mathrm{H}_{2} \mathrm{O}_{2}$ content in direct $\mathrm{H}_{2} \mathrm{O}_{2}$ scavenging with cFIR ${ }^{*} \mathrm{p}<0.05$ is significantly different compared to the control group. 


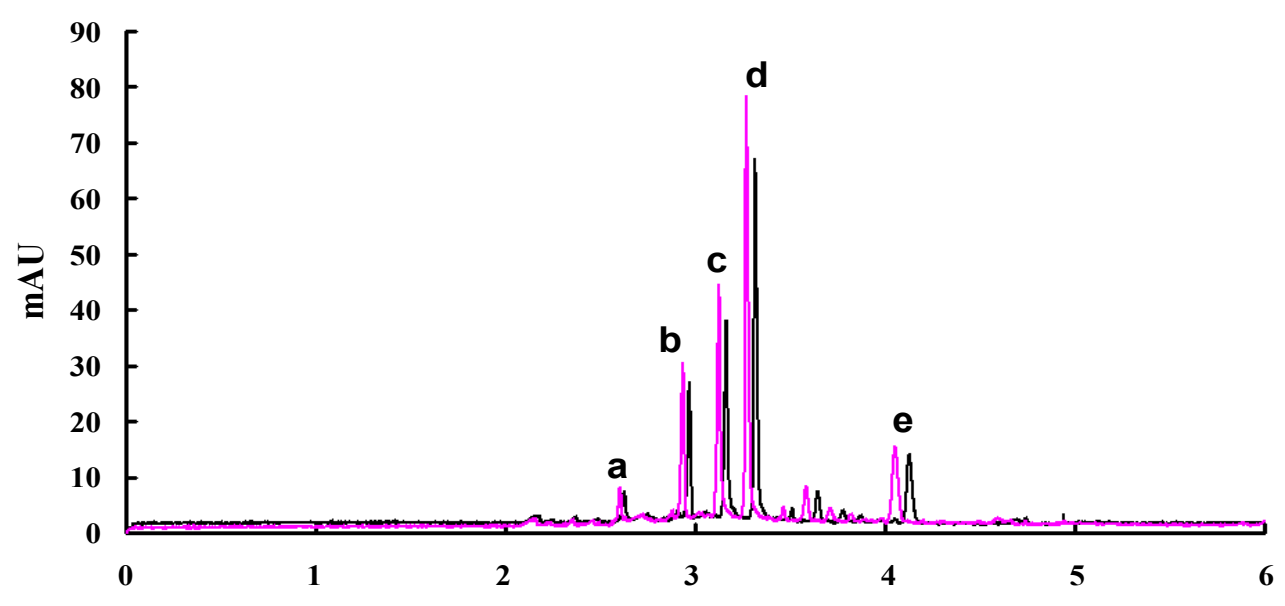


$c=E G C G$; $d=E G C$; $e=E C$. ( $Y$ axis: cumulative intensity $(A U)$ measured on chemicals pass; $X$ axis: running per minute).

In our study, we used DSC to precisely measure the different phases of transition energy released or absorbed of the different water samples to prove our previous deduction that cFIR is capable of changing the physical properties of water.

During this study, generally, the temperature program for DSC analysis is designed such that the sample holder temperature increases linearly as a function of time. The reference sample should have a welldefined heat capacity over the range of temperatures to be scanned. Our result of DSC analysis on the water sample without cFIR treatment showed an obvious change of heat flow $(\Delta \mathrm{Hc}$; Table 3$)$ during the transit from the liquid phase (water) to the solid phase (ice).

\section{Changes in $\mathrm{pH}$ on weak acid solution following cFIR irradiation}

Samples of $10 \%$ acetic acid solution without and with cFIR irradiation were measured for $\mathrm{pH}$ values. The results showed $1.8612 \pm$ 0.55 (without cFIR) and $1.8458 \pm 0.54$ (with cFIR) respectively $(\mathrm{n}=24)$. The observed decrease in $\mathrm{pH}$ for the irradiated group is significantly higher than that for the control group (Figure 10a; $p<.05$ ).

\section{Discussion}

According to our observation, cFIR exhibits its biological and medical effects not necessary for liquid water [31]. However, since living tissues of plants or animals are composed of water, we deduce those benefits of cFIR should be directly or indirectly through water's effect.

IR spectroscopy is by far the most precise method to provide information on hydrogen bonding which has a significant influence on the peak shape and intensity, generally causing peak broadening and peak shifting to lower frequencies (red-shift). Unlike the water molecule having a very small moment of inertia on rotation in vapor phase which gives rise to rich combined vibrational-rotational spectra, the FI-IR rotational spectra for liquid water molecules tend to be restricted by hydrogen bonds. IR spectroscopy was generally considered to be able to provide only qualitative and semi-quantitative analyses of common samples. However, the development of reliable FTIR instrumentation and strong computerized data-processing capabilities have greatly improved; thus, modern infrared spectroscopy has been accepted as a reliable tool for quantitative analysis [32].
Since the FT-IR measurement for non-cFIR DDW and cFIR irradiated DDW were carried out in a liquid cell with the same 150 $\mu \mathrm{m}$-Teflon spacer, the intensities of absorption bands were linearly proportional to the molecular absorptivity which might be attributed to the influence of the cFIR irradiation. Our result indicates cFIR exhibiting hydrogen bond weakening effect. We also performed a series of experiments to demonstrate other physical and chemical characteristics of the hydrogen bond weakening effect and possible applications, listed as below:

\section{Viscosity}

Viscosity Changes in hydrogen bonding have particularly important effects on viscosity and diffusion, which are shown by the large changes occurring in normal water. Viscosity is particularly affected by the strengthening of water's hydrogen bonds, increasing 10 -fold. For example, with a value of $37^{\circ} \mathrm{C}$, the increase in hydrogen bond strength is only $8 \%$. While the Stokes-Einstein equation predicts that the diffusion coefficient of a solute will be inversely proportional to the viscosity of the solvent [33]. According to the results, viscosity decreased by the cFIR irradiation on DD water. In previous studies, the absorption of sound was proven to have a specific range of frequency and intensity in distilled water under various conditions of sample illumination, and treatment with a magnetic field and ultrasound. The difference in sound signal absorption in water under unequal conditions is explained by variations in viscosity because of changes in the structural state of water, in particular, the destruction or formation of clusters. A similar phenomenon is noted in cFIR under room temperature, without the necessity of electricity-supported instruments, such as ultrasounds and strong magnetic fields. In this viscosity study, a weakening effect of the cFIR on hydrogen bonds to achieve decreases in viscosity was shown [1].

\section{Water cluster frequencies/Size}

Other researchers have claimed that FIR rays can break hydrogen bonds ( $\mathrm{H}-\mathrm{O}$ bonds) by exciting, "stretching or bending," vibrations in the water clusters; they can also reduce the size of the water clusters $[7,8,16,18,19]$.

Hydrogen bonds are the weakest type of chemical bond and are easily broken by thermal energy. Previous researches considered hydrogen bonds broken if the bond length increases or the bond angle 
decreases $[7,8,16,18,19]$. The hydrogen bonding holds water molecules approximately $15 \%$ closer than if water were composed of simple liquid molecules interacting with each other through van der Waals forces. However, hydrogen bonds restrict the number of neighboring water molecules to approximately four, instead of the larger numbers found in simple liquids $[1,34-36]$.

\section{Volatility}

The hydrogen bond is one factor that determines volatility. Any liquid that possesses hydrogen bonds may evaporate more quickly if the hydrogen bonds are broken. The effects of the weakening or destruction of hydrogen bonds is a major event in the enhancement of liquid volatility and energy transfer in liquid water. Volatility is an important physical property, and changes in volatility of an alcoholwater mixture may reflect a transformation of physical properties that are independent of chemical changes [37]. In this study, we used the dynamic headspace solid-phase micro extraction (HS-SPME) and GC to determine quantitatively the changes in the volatility of cFIRirradiated sorghum wine and water. We conclude that the effects of cFIR irradiation on water molecules enhanced the volatility of the liquor solution by weakening the hydrogen bonds.

\section{Diffusion}

Diffusion of liquid varies inversely with viscosity; we found that molecular movements slow as viscosity increases. Several factors determine the rate of diffusion of a molecule across the membrane depending on the size, polarity, and charge of each particular molecule. For an uncharged polar molecule to leave the aqueous phase and enter the lipid phase, it must first break its hydrogen bonds with water before it can dissolve in the lipid phase. The number of hydrogen bonds a molecule forming to water is determined by the number of polar groups in the molecule, and the strength of the hydrogen bonds formed. Each additional hydrogen bond formed between a polar group and water results in a 40-fold decrease in the partition coefficient, and a resulting decrease in the molecular permeability through the cell membrane [38].

\section{Surface tension (contact angle)}

The surface tension and viscosity of water are related to the strength of the hydrogen bonds between water molecules. The unusually large heat capacity of water is also related to the strength of the hydrogen bonds ( $\mathrm{H}-\mathrm{O}$ bonds) between water molecules that determine the characteristics of high volatility and viscosity. The decrease of volatility in the alcohol-water mixture of our cFIR treated samples is a reflection of decreased surface tension. Water droplets are strongly attracted to solid surfaces if the droplet spreads out completely on the solid surface and the contact angle is decreased. The weaker hydrophilic form has a higher contact angle of up to $90^{\circ} \mathrm{C}$.

Conversely, if a liquid is highly hydrophobic (less hydrophilic), the contact angle is larger. Because the water contact angle is proportionate to the water surface tension, a smaller water contact angle may be reflected by decreased surface tension [39]. Surface tension is caused by hydrogen bonds in the water sample holding tightly to one another and creating a type of structural rigidity $[39,40]$. Our results showed a significant reduction in the water contact angle of cFIR -treated water that was lower than the pretreated water (control group). Therefore, we deduce that cFIR material may induce water and related water mixtures to become lower in hydrophobicity and in lower surface tension, and perhaps weaken the hydrogen bonds. Particularly, this is the first study to show that the cFIR treated water can change the water contact angle.

\section{Hydrogen peroxide dissociation}

Previous studies have demonstrated that the thermal FIR treatment has the capability of cleaving covalent bonds to liberate more antioxidant compounds, for example, carotene and polyphenols from rice hulls and medicinal plants. Therefore, the increased heat transfer efficiency of FIR-emitting ceramic material explains the direct and indirect effects on $\mathrm{H}_{2} \mathrm{O}_{2}$-scavenging capacity. In addition, the FIR can break hydrogen bonds ( $\mathrm{H}-\mathrm{O}$ bonds) by exciting the stretching vibrations in the water clusters, and decreases the size of the water clusters [37].

Hydrogen bonds can reduce the volatility of any liquid possessing hydrogen bonds, and are related to the reduction rate of $\mathrm{H}_{2} \mathrm{O}_{2}$, which exist in cluster forms in normal conditions, and not as a single molecule. Therefore, the weakening of hydrogen bonds by FIR may also explain why the FIR accelerated the $\mathrm{H}_{2} \mathrm{O}_{2}$ transformation, and released $\mathrm{H}_{2} \mathrm{O}$ and $\mathrm{O}_{2}$ from $\mathrm{H}_{2} \mathrm{O}_{2}$ molecular clusters.

\section{Solubility of solid particles}

We showed that the FIR treatment at room temperature had significant effects on the water used to prepare the green tea. The total phenol contents, each major constitute concentration, and antioxidant activities of green tea were all enhanced by the FIR treatment. The three experiments in this study corresponded with each other, and indicate that more available or soluble catechins contribute to promoting the antioxidant activity of the green tea. No obvious difference was found in catechin degradation between the standard solutions dissolved by the FIR-treated water and control water. This indicates that the FIR effect on increasing catechin contents by enhancing the extraction efficiency on the green tea leaves instead of preventing the degradation of catechins is major. This shows that the cFIR causes the destruction of water clusters, and then facilitates water in extracting more functional constitutes of green tea leaves, and hence, enhances the antioxidant activities of the green tea. The rate-determining steps in tea leaf infusion were determined by the diffusion of the solutes through the leaf matrix to the surface. Destroyed water clusters increase the total number of water clusters, provide larger specific surfaces, and raise more contact opportunities for water molecules and green tea leaves, to extract more solutes in the green tea infusion.

\section{Water crystallization}

The results of our DSC experiment suggest that the increase in the freezing temperature observed for the cFIR-irradiated water may be caused by energy transfer in the water cluster through the influence of hydrogen bonds, creating an environment less conducive to the solid state $[38,39]$. The weakening effect of cFIR on the hydrogen bond likely accelerates water molecules and allows them to form hexagonal ice crystals [1].

\section{pH change on weak acid}

Acetic acid $\left(\mathrm{CH}_{3}-\mathrm{COOH}\right)$ possesses the hydrogen $(\mathrm{H})$ atom in the carboxyl group $(-\mathrm{COOH})$, which gives off an $\mathrm{H}^{+}$ion that produces its acidic character. Acetic acid is a weak acid, and hydrogen bonding effectively affects its acidity (Figures 11a and 11b). We deduced that the weakening of the intra-molecular hydrogen bond of the carboxyl group in acetic acid affected by the cFIR irradiation resulted in an increase in acidity [40].

\section{Future application and researches}

Water is the major constituent of living systems, such as the intracellular, extra cellular, and the circulation system. The influence 
Citation: Leung TK, Lin SL, Yang TS, Yang JC, Lin YS (2014) The Influence of Ceramic Far-Infrared Ray (cFIR) Irradiation on Water Hydrogen Bonding and its Related Chemo-physical Properties. Hydrol Current Res 5: 174. doi:10.4172/2157-7587.1000174

Page 9 of 10

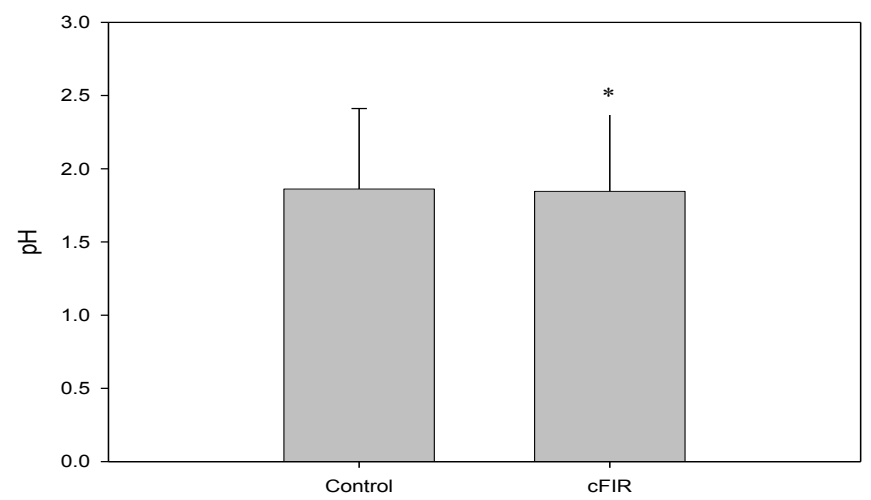

Figure 11a: Figure $\mathrm{f}$ Change of $\mathrm{PH}$ value of acetic acid, measurement by $\mathrm{PH}$ meter $(P$ value $<0.05, n=24)$;

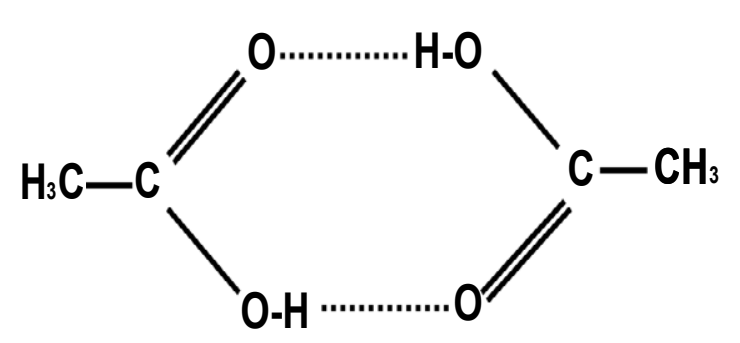

Figure 11b: In the solution of acetic acid, the intermolecular hydrogen bonds affect the abilities of the carboxyl group to ionize and behave as an acid. It is reflected by the direct function of the electronic properties and bonding orders of the atoms that comprise the carboxyl $(\mathrm{COOH})$ moiety.

of the cFIR on liquid water is probably a key explanation to the mysterious function of this specific spectrum of wavelengths toward living organisms. In the future, the cooperation of medical-biological and physical-chemical researchers is necessary for an in-depth study on this interesting topic.

We speculated that this phenomenon may be a consequence of a hydrogen bond-weakening effect and the unique 3D dynamic structural fluctuations and rearrangements of the hydrogen bonding network of water, which undergoes ultrafast structural reorientation and reorganization.

Because cFIR materials work on the "weakening of hydrogen bonds", the impact of this phenomenon contributes to different industrial applications relevant to its characteristics (Table 2).

\section{Conclusion}

Wedemonstrated theinfluence of room temperaturecFIRirradiation weakening hydrogen bonds by using quantitative measurements of viscosity, water cluster size/frequency, volatility, diffusion, surface tension (contact angle), hydrogen peroxide dissociation, solubility of solid particles, temperatures of water crystallization, changes in the $\mathrm{pH}$ of acetic acid, and the Fourier transform infrared spectroscopy (FT-IR) to produce physical and chemical changes in aqueous samples. Our methods tried various ways to demonstrate the weakening changes of hydrogen bonds by the cFIR irradiation. These physical effects may be one of the initial physical-biological mechanisms we observed in our previous medical-biological studies [13-22]. In the future, we wish to study further on potential applications of this effect.

\section{Acknowledgment}

The authors gratefully acknowledge the support provided to this study by Ms. Francis Chou (Hou Kunt Biological Technology CO.LTD), Mr. Ping Tai Lin (Leader Culture Co.), Mr. Francis Chen (Franz Collection, Taipei, Taiwan), and Mr. Li Chien Chiu (Hocheng---HCG, Taipei, Taiwan).

\section{References}

1. Chaplin MF (2000) A proposal for the structuring of water. Biophys Chem 24: 211-221.

2. Matsushita K (1988) Evaluation of the state of water by NMR spectrometry FIR Joho 5: 6-10.

3. Suresh SJ, Naik VM (2000) Hydrogen bond thermodynamic properties of water from dielectric constant data. J Chem Phys 113: 9727.

4. Yamaguchi Y, Yasutake N, Nagaoka M (2001) Theoretical prediction of proton chemical shift in supercritical water using gas-phase approximation. Chem Phy Letters 25: 129-136.

5. Luzar A, Chandler D (1996) Effect of Environment on Hydrogen Bond Dynamics in Liquid Water. Phy Rev Letters 76: 928-931.

6. Yokono T, Shimokawa S, Yokono M, Hat-tori H (2009) Infra-red spectroscopic study of structural change of liquid water induced by sunlight irradiation. WATER 1: 29-34.

7. LoparoJJ, Roberts ST, Tokmakoff A (2006) Multidimensional infrared spectroscopy of water Vibrational. I. dynamics in two-dimensional IR line shapes J Chem Phys 125: 194521.

8. Ashihara S, Huse N, Espagne A, Nibbering ETJ, Elsaesser T (2007) Ultrafast Structural Dynamics of Water Induced by Dissipation of Vibrational Energy. J Phys Chem A 111: 743-746.

9. Gragson DE, Richmond GL (1998) Investigations of the Structure and Hydrogen Bonding of Water Molecules at Liquid Surfaces by Vibrational Sum Frequency Spectroscopy. J Phys Chem B 102: 3847-3861.

10. Buch V, Milet A, Vácha R, Jungwirth P, Devlin JP (2007) Water surface is acidic PNAS 104: 7342-7347.

11. Leung TK, Yang JC, Lin YS (2012) The Physical, Chemical and Biological Effects by Room Temperature Ceramic Far-infrared Ray Emitting Material Irradiated Water: A Pilot Study J Chinese Chemical Soc 59: 589-597.

12. Ludwig $R$ (2002) The effect of hydrogen bonding on the thermodynamic and spectroscopic properties of molecular clusters and liquids. Phys Chem Phys 4: $5481-5487$.

13. Shigezo S, Tetsuro Y, Tadahiko M, Hiroki T, Tomoki E, et al. (2004) Effect of far-infrared light irradiation on water as observed by $\mathrm{X}$-ray diffraction measurements. Jpn J Appl Phys 43: L545-L547.

14. Lin SL, Chan WP, Choy CS , LeungTK (2013) Enhancement of Transderma Delivery of Indomethacin and Tamoxifen byFar-Infrared Ray-Emitting Ceramic Material (BIOCERAMIC): A Pilot Study. Transl Med 3: 1.

15. Lin SL, Choy CS, Chan WP, LeungTK (2013) Photoluminescence of Bioceramic Materials (PLB) as a Complementary and Alternative Therapy for Diabetes. Diabetes Metab 4: 10.

16. Leung TK, Lee CM, Lin MY(2009) Far infrared ray irradiation induces intracellular generation of nitric oxide in breast cancer cells. Journal of Medical and Biological Engineering 29: 15-18.

17. Leung TK, Lin YS, ChenYC (2009) Immuno modulatory effects of far-infrared ray irradiation via increasing calmodulin and nitric oxide production in raw 264.7 macrophages Biomedical Engineering. 21: 317-323.

18. Leung TK, Lee CM, Tsai SY, Chen YC, Chao JS (2011) A pilot study of ceramic powder far-infrared ray irradiation(cFIR) on physiology: observation of cell cultures and amphibian skeletal muscle. Chin J Physiol 54: 247-254.

19. Leung TK, Lin YS, Lee CM (2011) Direct and indirect effects of ceramic far infrared radiation on hydrogen peroxidescavenging capacity and on murine macrophages under oxidative stress. Journal of Medical and Biological Engineering 31: 345-351.

20. Leung $\mathrm{TK}$, Chen $\mathrm{CH}$, Lai $\mathrm{CH}$ (2012) Bone and joint protection ability of ceramic material with biological effects. The Chin J Physiol 55: 47-54.

21. Leung TK, Chen CH, Tsai SY, Hsiao G, Lee CM (2012) Effects of far infrared rays irradiated from ceramic material (bioceramic) on psychological stress- 
Citation: Leung TK, Lin SL, Yang TS, Yang JC, Lin YS (2014) The Influence of Ceramic Far-Infrared Ray (cFIR) Irradiation on Water Hydrogen Bonding and its Related Chemo-physical Properties. Hydrol Current Res 5: 174. doi:10.4172/2157-7587.1000174

conditioned elevated heart rate, blood pressure, and oxidative stresssuppressed cardiac contractility. Chin J Physiol 55: 323-330.

22. Leung TK, Lin JM, Chien HS, DayTC (2012) Biological effects of melt spinning fabrics composed of $1 \%$ bioceramic material. Textile Research Journal 82 $1121-1130$

23. Leung TK, Chan CF, Lai PS, Yang CH, Hsu CY, et al. (2012) Inhibitory effects of far-infrared irradiation generated by ceramic material on murine melanoma cell growth. Int J Photoenergy.

24. Leung TK, Liu YC, Chen $\mathrm{CH}$, Fang HN, Chen KC, et al. (2012) In vitro cell study of the possible anti-inflammatory and pain relief mechanism of far-infrared ray emitting ceramic material. Journal of Medical and Biological Engineering 33: 179-184

25. Chen CW, Tai CJ , Choy CS, Hsu CY, Lin SL, et al. (2013) Wave-Induced Flow in Meridians Demonstrated Using Photoluminescent Bioceramic Material on Acupuncture Points. Evidence-Based Complementary and Alternative Medicine Volume 2013

26. Cheng CM, Chang MC, Chang YF, Wang WT, Hsu CT, et al. (2012) Optical Tweezers-Assisted Cross-Correlation Analysis for a Non-intrusive Fluid Temperature Measurement in Microdomains. Jpn J Appl Phys 51: 067002.

27. Tongcang L, Kheifets S, Medellin D, Raizen MG (2010) Measurement of the Instantaneous Velocity of a Brownian Particle. Science 328: 1673-1675.

28. Djordjevic J, Michniak B, Uhrich KE (2003)Amphiphilic star-like macromolecules as novel carriers for topical delivery of nonsteroidal anti-inflammatory drugs. AAPS Pharm Sci 5: E26.

29. Labata L, Deveauxb M, Dalleta P, Dubosta JP (2002) Separation of new antidepressants and their metabolites by micellar electrokinetic capillary chromatography. J Chromatogr B Analyt Technol Biomed Life Sci 773: 17-23.

30. Leung TK, Huang PJ, Chen YC, Lee CM (2011) Physical chemical test platform for room temperature, far-infrared ray emitting ceramic materials (cFIR). J Chin Chem Soc 58: 653-658.
31. Martens H, Bruun SW, Adt I, Sockalingum GD, Kohler A (2006) Pre-processing in biochemometrics: correction for path-length and temperature effects of water in FTIR bio-spectroscopy by EMSC. J Chemometrics 20: 402-417.

32. Su JT, Duncan PB, Momaya A, Jutila A, Needham D (2010) The effect of hydrogen bonding on the diffusion of water in n-alkanes and n-alcohols measured with a novel single microdroplet method. J Chem Phys 132: 044506.

33. Cho $\mathrm{CH}$, Urquidi J, Robinson GW (1999) Molecular level description of temperature and pressure effects on the viscosity of water. J Chem Phys 111 10171-10176.

34. Vedamuthu M, Singh S, Robinson GW (1994) Properties of liquid water: origin of the density anomalies. J Chem Phys 98: 2222-2230.

35. Peeters DJ (1995) Hydrogen bonds in small water clusters: A theoretical point of view. J Mol Liq 67: 49-61.

36. Krusic PJ, Marchione AA, Davidson F, Kaiser MA, Kao CPC, et al. (2005) Vapo Pressure and Intra-molecular Hydrogen Bonding in Fluorotelomer Alcohols $\mathrm{J}$ Phys Chem A 109: 6232-6241.

37. Eckert R (1998) Permeability and transport; in Animal physiology, (3rdedn): W H. Freeman, New York

38. Giovambattista N, Rossky PJ, Debenedetti PG (2007) Effect of Temperature on the Structure and Phase Behavior of Water Confined by Hydrophobic, Hydrophilic, and Heterogeneous Surfaces. J Phys Chem B 111: 9581-9587.

39. Luzar S (1983) The contribution of hydrogen bonds to the surface tension of water. Chem Phys Lett 96: 485-490.

40. Lee SC, Kim JH, Jeong SM, Kim DR, Ha JU, et al. (2003) Effect of Far-Infrared Radiation on the Antioxidant Activity of Rice Hulls. J Agric Food Chem 51: 4400 4403. 